\title{
Topographic Analysis of Flash Visual Evoked Potentials in Dogs
}

\author{
Tomofumi KIMOTSUKI ${ }^{1,2)}$, Mitsuya YASUDA ${ }^{1)}$, Satoshi TAMAHARA ${ }^{2)}$, Naoaki MATSUKI') and Kenichiro ONO ${ }^{2) *}$ \\ ${ }^{1)}$ Medicinal Safety Research Laboratories, Sankyo Co., Ltd., 717 Horikoshi, Fukuroi, Shizuoka 437-0065 and ${ }^{2)}$ Department of Veterinary \\ Clinical Pathobiology, Graduate School of Agricultural and Life Sciences, The University of Tokyo, 1-1-1 Yayoi, Bunkyo-ku, Tokyo 113- \\ 8657, Japan
}

(Received 20 August 2004/Accepted 10 May 2005)

ABSTRACT. Visual evoked potentials by flash stimulation (flash VEP) were analyzed in dogs using a topographic method. The flash VEP consisted of 3 positive (P1, P2 and P3) and 2 negative (N1 and N2) components by $150 \mathrm{msec}$ after the flash stimuli. On the topographic mappings, a negative response area was observed in the frontal region of the scalp in the stimulated site followed by the shifting of the area to the contralateral frontal region and occipital region, during the first $100 \mathrm{msec}$. The negative response area in the frontal region in the stimulated site, contralateral frontal and temporal region, and occipital region were corresponded to N1, P2, and N2 on the flash VEP, respectively, according to their latencies. In the dogs with experimentally impaired the right lateral geniculate body, the latency of $\mathrm{P} 2$ was prolonged, and $\mathrm{N} 2$ and $\mathrm{P} 3$ were disappeared after the left eye stimulation. On the topographic mapping, only the early negative response area was detected on the stimulated site of the frontal region of the brain. Therefore, it is concluded that P1 and N1, P2, and $\mathrm{N} 2$ are referred to the retinal potentials, the potentials from the retina to the brainstem included the lateral geniculate body, and those from the brainstem to the visual cortex, respectively.

KEY WORDS: canine, flash stimulus, lateral geniculate body, topographic mapping, visual evoked potential.

J. Vet. Med. Sci. 67(9): 869-875, 2005

Visual evoked potentials (VEPs) indicated electrophysiological response from retina to visual cortex is one of the clinical diagnostic techniques in human medicine to evaluate for the central nervous disorders, especially optic pathway disturbance. The flash VEP stimulated by the flashlight has also been recorded in dogs $[1,5,6,9-11]$, however the generators of potentials in amplitude and/or latency of the waves, are not well known. Recently, topographic analysis of evoked potentials have been applied in human using multiple electrodes on the scalp for elucidating generators of the waves in the flash VEP $[3,7,8,12]$. The topographic maps have also been used for evaluation of central nervous disorders, since the distribution of potentials recorded on the scalp in a selected time is visualized two-dimensionally in this method $[7,13]$. On the other hand, VEP is reported to be a sensitive indicator for dementia in human, especially Alzheimer's disease. Visser et al. [14] demonstrated that the latency of the flash VEP component was delayed in the patients with dementia. As there are few diagnostic techniques for dementia in dogs, the flash VEP was evaluated using Beagle dogs.

In this study, the flash VEP were recorded in beagle dogs after dark adaptation and topographic analysis was carried out on the scalp potentials using multiple electrodes. In addition, the changes of these potentials and maps were evaluated in dogs with an experimentally impaired the lateral geniculate body, which is critical intermediate nucleus of the optic pathway in the brain.

\footnotetext{
* Correspondence to: Ono, K., Department of Veterinary Clinical Pathobiology, Graduate School of Agricultural and Life Sciences, The University of Tokyo, 1-1-1 Yayoi, Bunkyo-ku, Tokyo 113-8657, Japan.
}

\section{MATERIALS AND METHODS}

Animals: Three male and 7 female beagle dogs aged from 1 - to 3-year-old were used for the flash VEP and topographic mapping. Other 2 male and 4 female same aged beagle dogs were used for the experimentally impaired the right lateral geniculate body by the heating technique. This study was approved by the Institutional Animal Care and Use Committee of Sankyo Co., Ltd., Tokyo, Japan.

The flash VEP: The flash VEP was recorded using needle electrodes inserted in the midline of the scalp according to the method reported by Strain et al. [10]. The electrodes of recording, reference, and ground were placed over the midline of the nuchal crest $(\mathrm{Oz})$, forehead $(\mathrm{Fpz})$ and vertex, respectively (Fig. 1-A). Prior to recording, the dogs were instilled the mydriatic drug including tropicamide and phenylephrine hydrochloride, and subcutaneously given atropine sulfate $(0.05 \mathrm{mg} / \mathrm{kg})$ and intramuscularly given xylazine $(2-3 \mathrm{mg} / \mathrm{kg}$ ) for sedation. Then the dogs were dark-adapted at least $1 \mathrm{hr}$ and the flash VEP were recorded in the dark shielded room. The white flash stimulus was given $1 / \mathrm{sec}$ with the intensity of 0.6 joule using a photostimulator (SLS-3100 Photostimulator, Nihon Kohden Co., Tokyo, Japan). The xenon lamp was located about $15 \mathrm{~cm}$ in the front of the examined eye and another eye was covered with the black cloth. The responses were recorded with signal averager (Neuropack Four Mini, Nihon Kohden Co., Tokyo, Japan). The bandpass filter was set at $1 \mathrm{~Hz}$ low and $1,000 \mathrm{~Hz}$ high. In this study, consecutive 30 responses were averaged and the latency and amplitude of the flash VEP components were measured.

Topographic mapping: The arrangement of electrodes on the scalp was as follows: The scalp area was divided with 

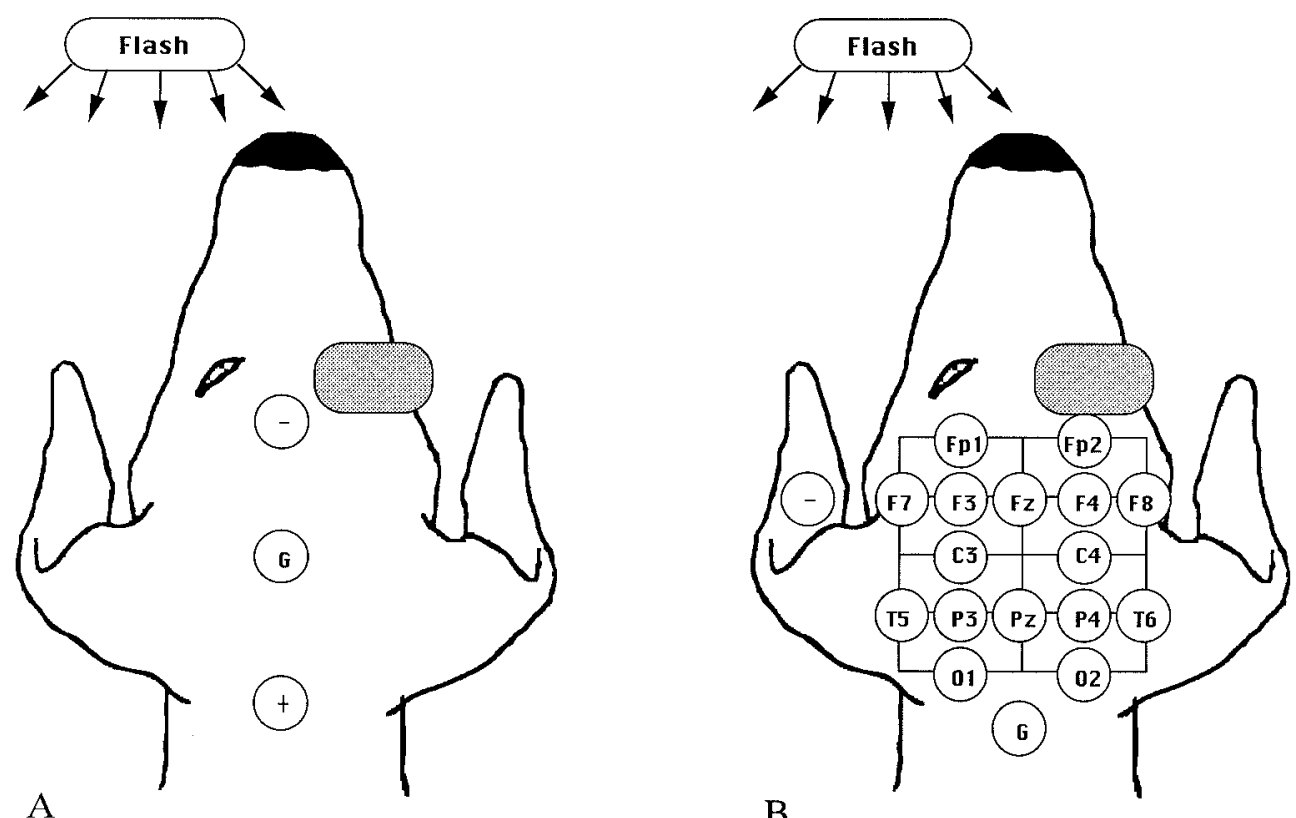

Fig. 1. Arrangement of electrodes on the scalp for recording the flash VEP (A) and topographic mapping (B).

matrix consisted of $5 \times 5$ matrices. Electrodes were placed on sixteen of them. The distance of each electrode between right and left, and back and forth was 15 and $10 \mathrm{~mm}$, respectively (Fig. 1-B). The abbreviations of electrodes were referred to the 10-20 electrodes using system in human [4]. The potentials of the residual matrices were calculated from adjacent potentials of electrode. After shaving the scalp, the disk electrodes fixed on a cloth to maintain the location of electrodes were fastened on the scalp with an elastic tubular bandage. The reference and ground electrode was placed on the stimulated ipsilateral ear and the neck, respectively. The responses were amplified and stored by data acquisition system (MP100, BIOPAC Systems Inc., Santa Barbara, California). Topographic maps were constructed from the averaging of the 30 responses obtained from each electrode using an evoked potential analyzer (GENIUS, Medical Research Equipment Co., Tokyo, Japan).

Experimentally impaired the right lateral geniculate body: The right lateral geniculate body in the dog's brain was impaired by the method of electric current of high frequency using a lesion generator (RFG-4A Radio Frequency Lesion Generator, Radionics Inc., Burlington, Massachusetts). Briefly, the dog was anesthetized with xylazine hydrochloride (2-3 mg/kg, i.m.), ketamine hydrochloride $(10 \mathrm{mg} / \mathrm{kg}$, i.v.) and atropine sulfate $(0.05 \mathrm{mg} / \mathrm{kg}$, s.c.). After the intubation of an endotracheal tube, anesthesia was maintained with halothane. The head of the dog was secured in the stereotaxic headholder to keep the base of the brain horizontally. The scalp was shaved, sterilized, and incised and the right temporal muscle was peeled. Holes of $2 \mathrm{~mm}$ in diameter that located $25-35 \mathrm{~mm}$ forward from inion and $10-15 \mathrm{~mm}$ right from midline were made on the cranial bone using a dental instrument. The heating electrode was vertically introduced $30-35 \mathrm{~mm}$ in depth into the brain from the hole. The tissue adjacent to the tip was maintained $80^{\circ} \mathrm{C}$ for 3 min under the control of current flow. After the heating, the scalp was replaced and antibiotics were administered for 4-5 days. The treated animals were in clinically healthy in food intake, activity and gait until autopsy. On electroretinogram (ERG) recorded in the treated dogs, no significant changes were observed after both sides of eye stimulation (data not shown). The flash VEP was recorded at pre, 2 and 21 days after the treatment. After the experiment, the dogs were euthanized by intravenous injection with sodium pentobarbital followed by exsanguination. The part of the lesion was assured in the brain of each dog. On the histological examination, fusion and necrosis were observed at the right lateral geniculate body area in the brain (data not shown).

\section{RESULTS}

The flash VEP: The flash VEP consisted of 3 positive and 2 negative components was recorded by $150 \mathrm{msec}$ after the flash stimuli. The mean latency of the P1, N1, P2, N2 and $\mathrm{P} 3$ peak was $15,31,53,81$, and $142 \mathrm{msec}$, respectively, in the averaged waveform in 10 dogs (Table 1 and Fig. 2).

Topographic mapping: The remarkable negative potential included oscillatory potentials was observed at the ipsilateral frontal electrodes (Fp1, F3 and F7) on the waveforms recorded from 16 electrodes on the scalp by $30 \mathrm{msec}$ after the left eye stimulation. The peak of negative potentials was detected on the contralateral frontal electrode (Fp2) at 40 $60 \mathrm{msec}$. The latency of the negative potential peak gradu- 
Table 1. Latency and amplitude of the components on the flash VEP waveform in dogs

\begin{tabular}{cccccccccc}
\hline \multicolumn{4}{c}{ Latency $(\mathrm{msec})$} & & \multicolumn{4}{c}{ Amplitude $(\mu \mathrm{V})$} \\
\cline { 1 - 6 } \cline { 7 - 9 } P1 & $\mathrm{N} 1$ & $\mathrm{P} 2$ & $\mathrm{~N} 2$ & $\mathrm{P} 3$ & & P1-N1 & N1-P2 & P2-N2 & N2-P3 \\
\hline 14.8 & 30.6 & 52.9 & 81.4 & 142.0 & & 25.9 & 25.2 & 27.1 & 36.3 \\
\pm 1.7 & \pm 5.1 & \pm 6.0 & \pm 7.6 & \pm 12.5 & & \pm 13.4 & \pm 11.4 & \pm 15.5 & \pm 16.8 \\
\hline
\end{tabular}

The values indicate mean $\pm \mathrm{SD}, \mathrm{n}=10$.

ally delayed from frontal to occipital electrodes (Fig. 3). Symmetrical responses were also observed in the right eye stimulation (data not shown). On the series of topographic mapping, a negative focus indicating as a blue area was found on the left frontal region at $30 \mathrm{msec}$ after the left eye

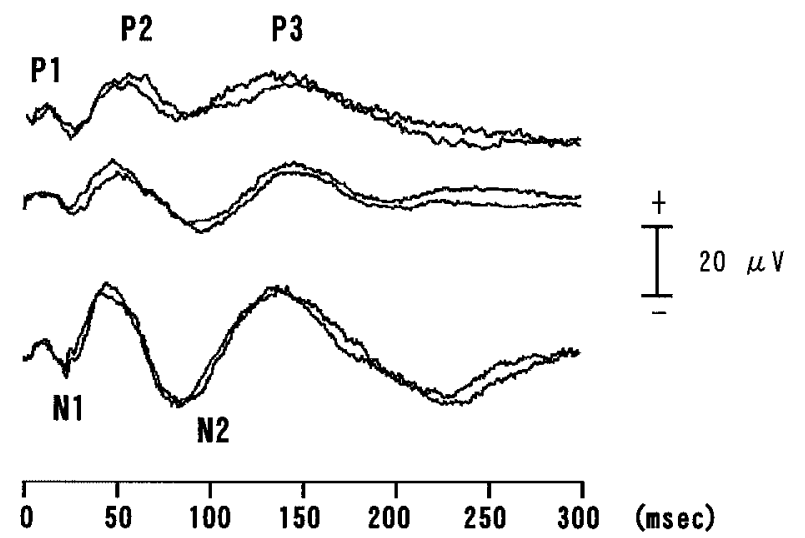

Fig. 2. Typical waveforms on the flash VEP in dogs. stimulation. The negative area moved to the contralateral frontal region at $40-60 \mathrm{msec}$ followed by the occipital region at $60-100 \mathrm{msec}$ after the stimuli (Fig. 4). The time of negative areas detected in the frontal region in the stimulated site, contralateral frontal and/or temporal, occipital, and frontal region on the topographic mapping was correspond with the latency of N1, P2, N2 and P3 on the flash VEP, respectively (Fig. 5).

Experimentally impaired the right lateral geniculate body: On the pretreatment, the flash VEP showed a typical waveform included 3 positive and 2 negative components in both right and left eye stimulation. However, disappearance of N2 and P3 was observed at 2 days after the treatment, and delay of P2 latency and disappearance of N2 and P3 were observed at 21 days after the treatment in the left eye stimulation. In contrast, all components were detected without changing of their latency in the right eye stimulation. Although the amplitude of the components was reduced in the right eye stimulation at 2 days after the treatment, they were recovered at 21 days after the treatment (Table 2, Fig.6). On the topographic mapping, the negative area remained on the left frontal region without moving to contralateral frontal and occipital region was observed by 70

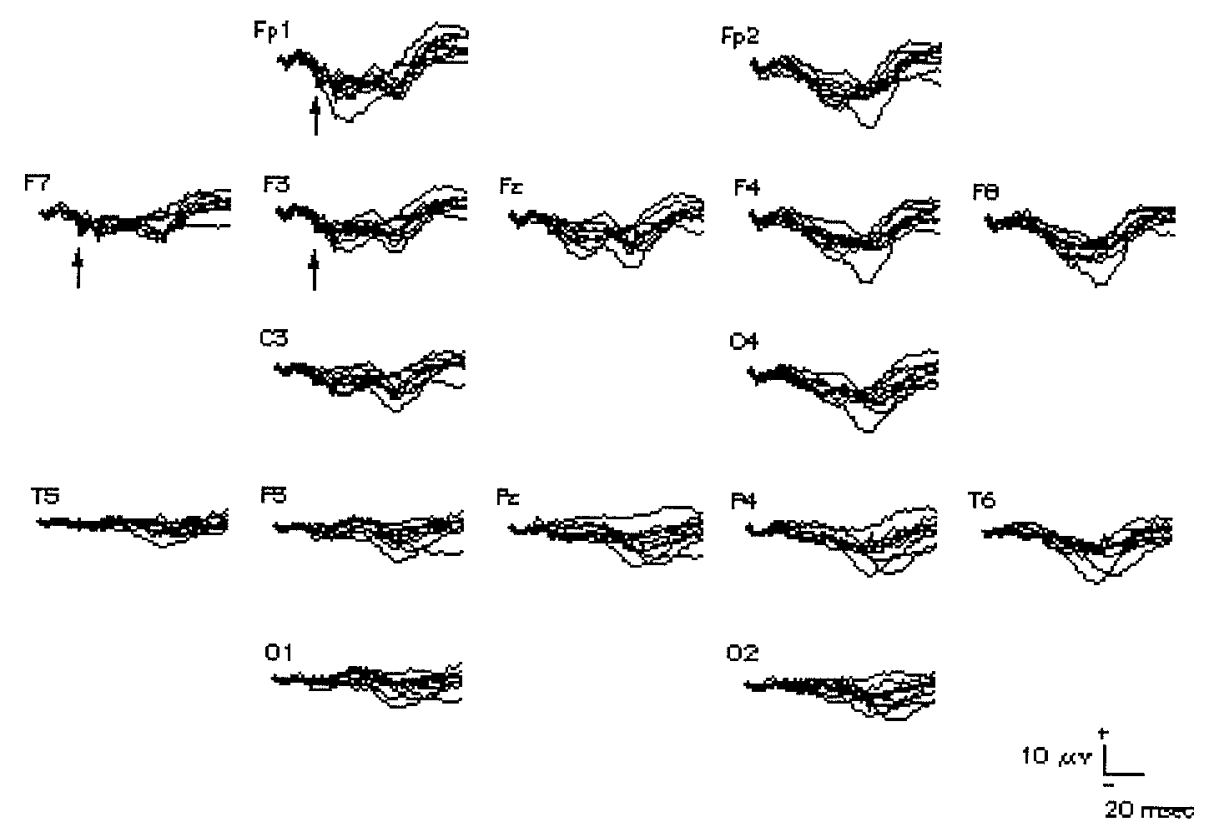

Fig. 3. VEPs recorded from 16 electrodes on the scalp by $100 \mathrm{msec}$ after the flash stimuli in 10 dogs. Arrows indicate oscillatory potentials according to the waveform and latencies. 


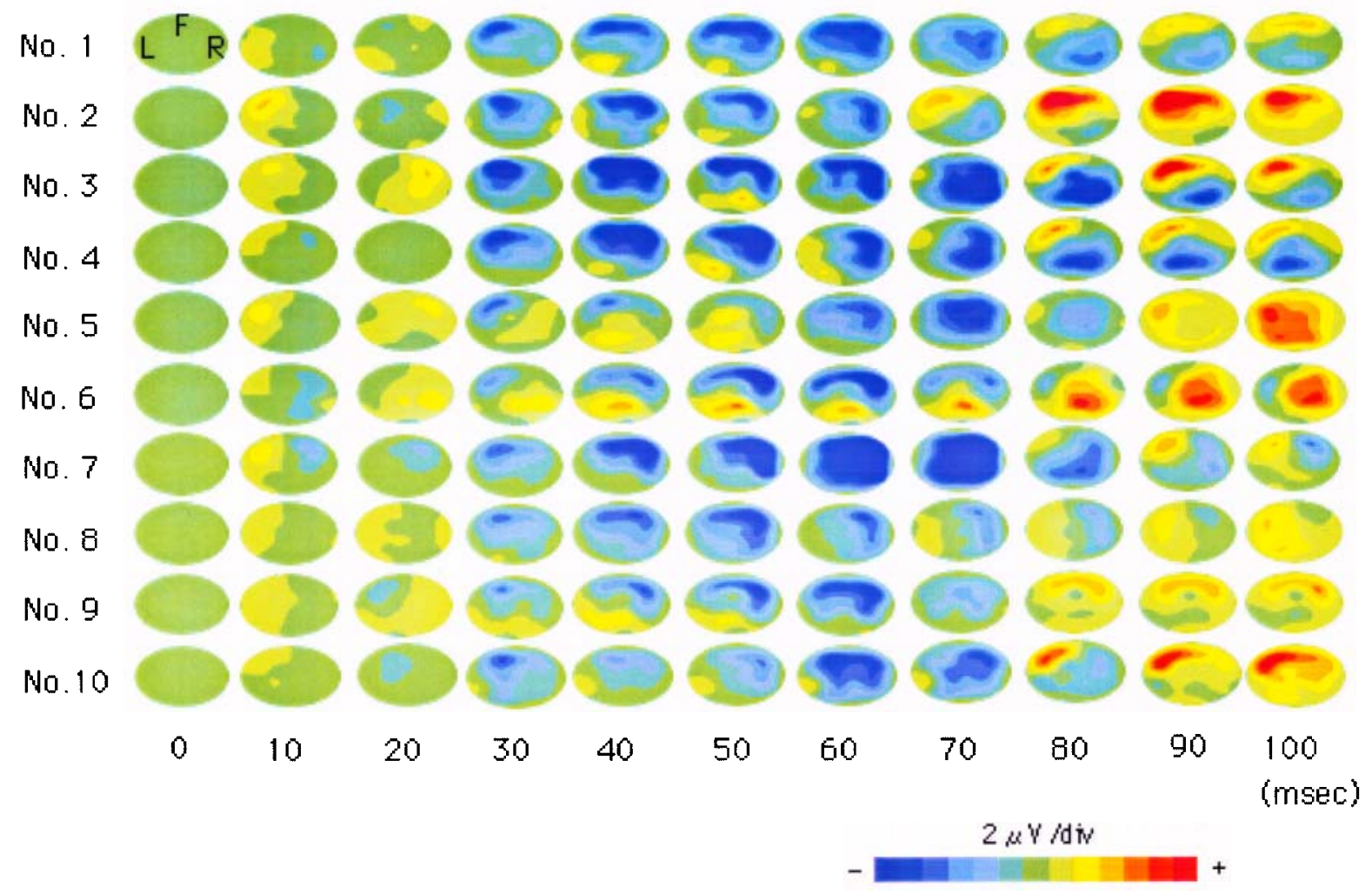

Fig. 4. Topographic mappings obtained from visual evoked potentials by $100 \mathrm{msec}$ after the flash stimuli in dogs. A negative focus indicating as a blue area was found on the left frontal region at $30 \mathrm{msec}$ after the left eye stimulation. The negative area moved to the contralateral frontal region at 40-60 msec, followed by the occipital region at 60-100 msec after the stimuli. In a picture of the topography at $0 \mathrm{msec}, \mathrm{F}, \mathrm{R}$, and $\mathrm{L}$ indicate front, right, and left on the scalp, respectively.

msec after the left eye stimulation at 21 days after the treatment (Fig. 7).

\section{DISCUSSION}

In this study, the topographic mapping of evoked potentials obtained from 16 electrodes on the scalp showed negative responses, moving from frontal to occipital region in the brain by $100 \mathrm{msec}$ after the flash stimuli. On the other hand, the flash VEP recorded between the forehead and the inion consisted of 3 positive and 2 negative components like as those reported previously [10].

The flash VEP was composed of 3 responses between 10 to $70 \mathrm{msec}$ after the stimuli in human [12]. The first response included 4 oscillatory potentials appeared on the frontal region from 10 to $40 \mathrm{msec}$ after the stimuli on the topographic mapping. As the second, a large positive potential followed by a weak negative one was observed on the central and vertex region from 50 to $57 \mathrm{msec}$. The third was parieto-occipital potentials, which corresponded to $\mathrm{N}_{70}$ of the flash VEP, appearing from 40 to $70 \mathrm{msec}$. These frontal, central, and occipital responses were reflected retinal potentials, brainstem optic pathway potentials, and occipital potentials, respectively. In this study, the course of responses after the flash stimuli in dogs was similar to those in human. Briefly, negative potentials including oscillatory potentials were observed in the waveforms recorded from electrodes located on the stimulated side by $30 \mathrm{msec}$ after the stimuli. On the topographic mapping, the earliest response was also detected as a negative area on stimulated frontal region at $30 \mathrm{msec}$ after the stimuli. This response was interpreted as retinal potentials, because the components of the waveform and their latency were coincident with a- and b-waves on the ERG [6]. Subsequent negative response area was observed on the contralateral side in the brain from 40 to $60 \mathrm{msec}$ and the occipital region from 60 to $100 \mathrm{msec}$ after the stimuli on the topographic map indicated the response from retina to brainstem and the potentials in the visual cortex, respectively. From the results of the flash VEP and topographic mapping, therefore, the latency of N1, P2 and N2 in the flash VEP was represented the time when negative response was noted stimulated frontal, contralateral frontal, and occipital region, respectively. The responses until N2 were considered to reflect the conduction of electrical signals from retina to visual cortex.

After the impairment of the right lateral geniculate body, the flash VEP showed a delay of P2 latency and disappearance of N2 and P3 in the left eye stimulation, whereas major 

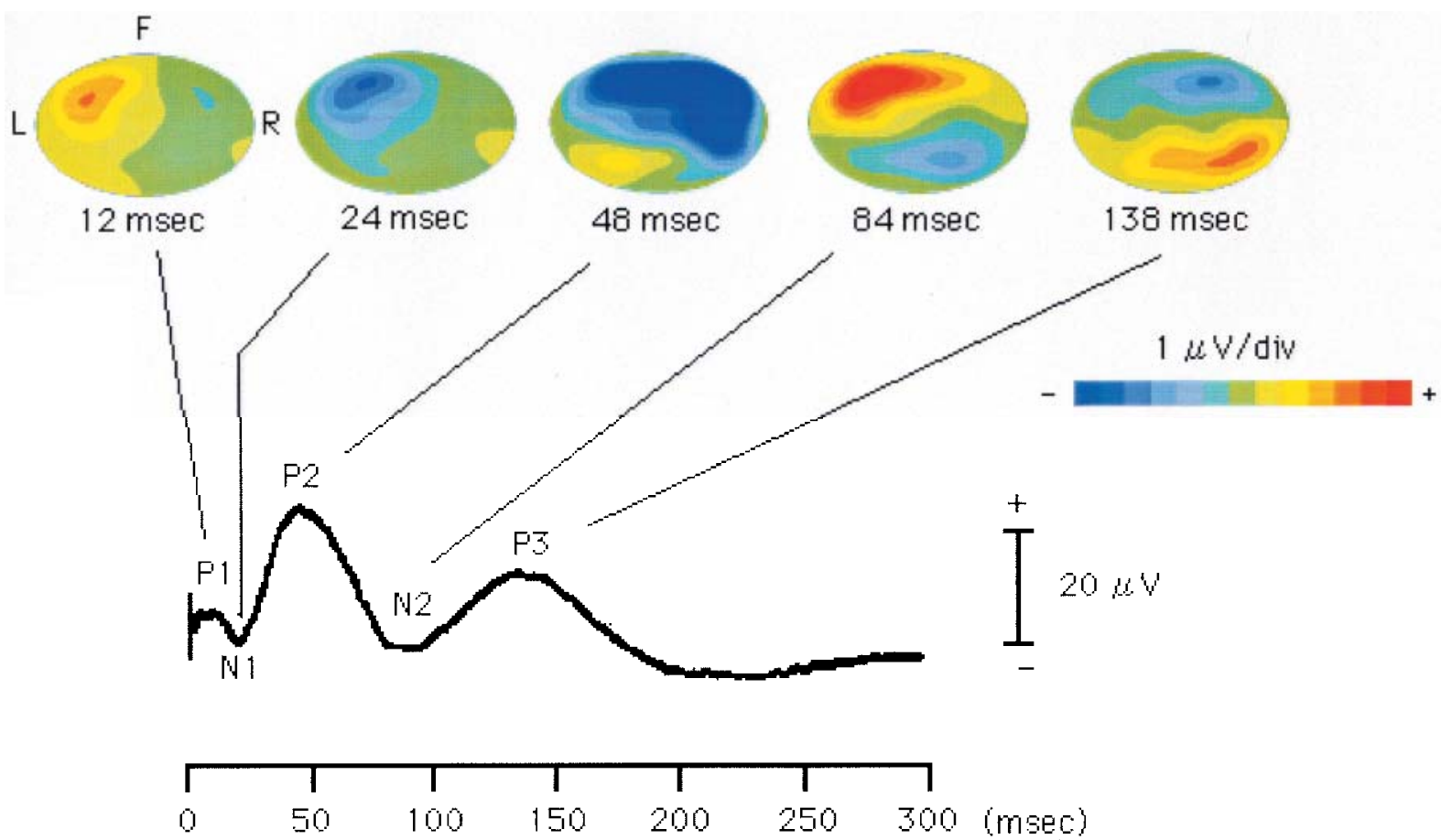

Fig. 5. Topographic mappings corresponding to the latency of each averaged waveform on the flash VEP. In a picture at $12 \mathrm{msec}, \mathrm{F}, \mathrm{R}$, and $\mathrm{L}$ indicate front, right, and left on the scalp, respectively.

Table 2. Latencies and amplitudes on the flash VEP waveform in dogs with heat impairment of the right lateral geniculate body

\begin{tabular}{|c|c|c|c|c|c|c|c|c|c|c|}
\hline & \multirow{2}{*}{$\begin{array}{c}\text { Days after } \\
\text { treatment }\end{array}$} & \multicolumn{5}{|c|}{ Latency (msec) } & \multicolumn{4}{|c|}{ Amplitude $(\mu \mathrm{V})$} \\
\hline & & $\mathrm{P} 1$ & N1 & $\mathrm{P} 2$ & N2 & P3 & $\mathrm{P} 1-\mathrm{N} 1$ & $\mathrm{~N} 1-\mathrm{P} 2$ & $\mathrm{P} 2-\mathrm{N} 2$ & N2-P3 \\
\hline \multirow{6}{*}{$\begin{array}{l}\text { Right eye } \\
\text { stimulation }\end{array}$} & Pre & 9.5 & 23.0 & 45.9 & 89.7 & 136.0 & 8.5 & 27.9 & 38.3 & 28.4 \\
\hline & & \pm 3.2 & \pm 1.3 & \pm 4.1 & \pm 4.1 & \pm 6.2 & \pm 4.7 & \pm 6.0 & \pm 7.3 & \pm 11.2 \\
\hline & 2 days & 9.8 & 27.1 & 50.8 & 83.5 & 135.0 & 12.3 & $17.9 *$ & $11.7 * *$ & 20.7 \\
\hline & & \pm 2.3 & \pm 10.3 & \pm 8.7 & \pm 11.6 & \pm 9.1 & \pm 8.7 & \pm 7.2 & \pm 6.7 & \pm 14.5 \\
\hline & 21 days & 9.3 & 23.6 & 48.4 & 84.8 & 136.0 & 8.8 & $20.8 *$ & $22.5^{*}$ & 25.3 \\
\hline & & \pm 2.8 & \pm 1.7 & \pm 4.3 & \pm 7.0 & \pm 11.6 & \pm 3.6 & \pm 3.6 & \pm 7.0 & \pm 9.6 \\
\hline \multirow{6}{*}{$\begin{array}{l}\text { Left eye } \\
\text { stimulation }\end{array}$} & Pre & 8.6 & 24.1 & 50.3 & 90.0 & 133.0 & 10.6 & 25.7 & 25.8 & 21.7 \\
\hline & & \pm 2.7 & \pm 1.4 & \pm 2.9 & \pm 4.6 & \pm 7.8 & \pm 4.4 & \pm 5.9 & \pm 9.2 & \pm 11.3 \\
\hline & 2 days & 8.6 & 23.1 & 52.0 & ND & ND & 6.9 & 17.3 & ND & ND \\
\hline & & \pm 2.8 & \pm 2.9 & \pm 11.3 & & & \pm 2.4 & \pm 3.2 & & \\
\hline & 21 days & 8.3 & 24.1 & $64.5 * *$ & ND & ND & 14.5 & 29.4 & ND & ND \\
\hline & & \pm 2.0 & \pm 1.3 & \pm 4.0 & & & \pm 3.4 & \pm 4.1 & & \\
\hline
\end{tabular}

The values indicate mean $\pm \mathrm{SD}, \mathrm{n}=6, \mathrm{ND}$ : not detected, $* \mathrm{p}<0.05, * *: \mathrm{p}<0.01$.

components were detected in the right eye stimulation. In dogs, optic nerve fibers predominantly project to the contralateral dorsolateral geniculate nucleus [2]. The marked changes of the flash VEP in the left eye stimulation were considered to reflect the anatomical disturbance of the visual pathway in the left eye. The delay of P2 latency and disappearance of N2 and P3 indicated that the generator of these potentials exist in the lateral geniculate bodies and/or behind them. The serial topographic mappings recorded from the treated dogs also demonstrated the obstruction in the visual pathway. The remaining of negative area indicated retinal potentials was observed on the frontal region of the stimulated side. The responses from the contralateral side to occipital region were not obviously observed in the left eye stimulation. Although decrease of amplitude of the components (N1-P2 and P2-N2) was observed in the flash VEP in the right eye stimulation at 2 days after the treatment, both of them were almost recovered at 21 days after the treatment. These changes suggested that some effects of the treatment on the visual pathway, especially contralateral 
Right eye stimulation

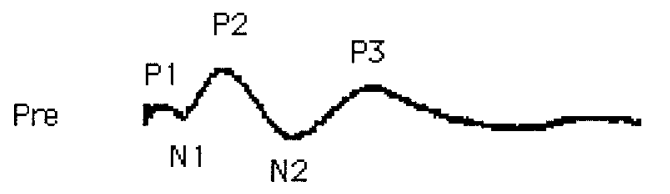

2 days

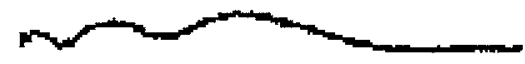

21 days
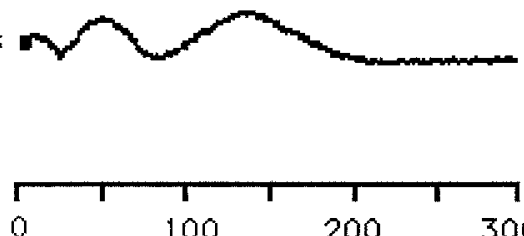

Left eye stmulation
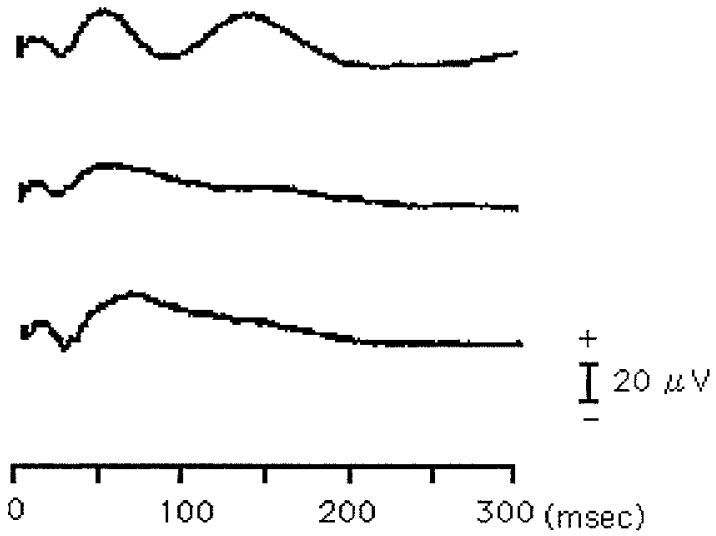

Fig. 6. Change of the flash VEP waveform after the heat impairment of the right lateral geniculate body. Delay of P2 latency, and disappearance of N2 and P3 were observed at 2 days and 21 days after the treatment, respectively, in the left eye stimulation. Each wave represents the average in 6 dogs.

Right eye stimulation

Pre
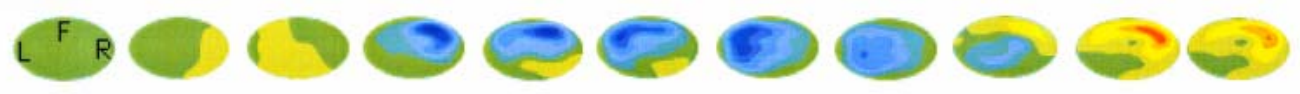

2 days
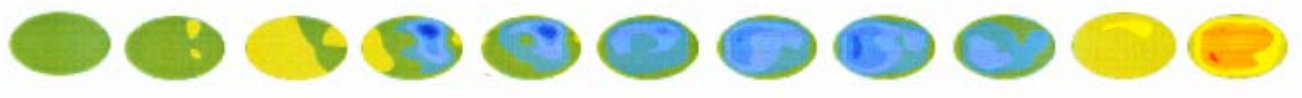

21 days
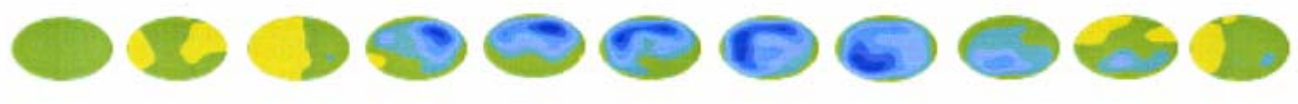

Left eye st mulation

Pre
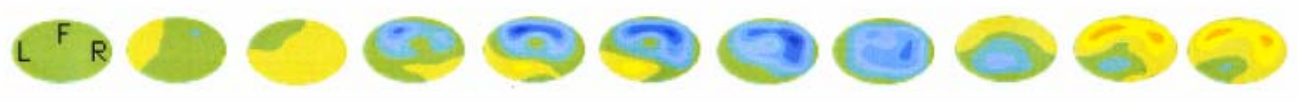

2 days
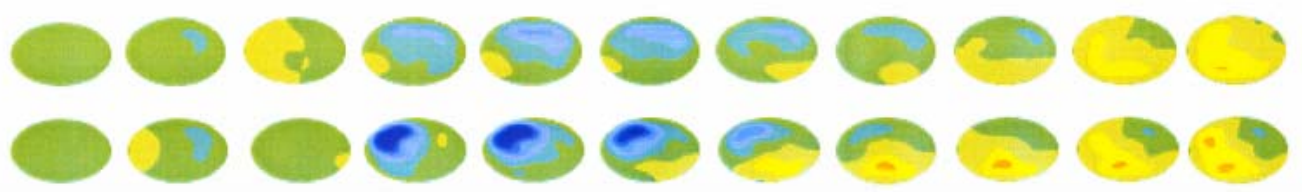

0

20

30

40

50

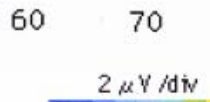

80
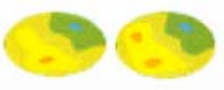

100

(msec)

Fig. 7. Averaged topographic mappings of the flash VEP in dogs with heat impairment of the right lateral geniculate body. The negative area remained on the left frontal region without moving to contralateral frontal and occipital region was observed by $70 \mathrm{msec}$ after the left eye stimulation at 21 days after the treatment. In a picture at $0 \mathrm{msec}, \mathrm{F}, \mathrm{R}$, and $\mathrm{L}$ indicate front, right, and left on the scalp, respectively.

geneculate body (right lateral genaculate body) impaired, in which passed through small part of retinal potentials.

From these results, it is concluded that P1 and N1, P2, and $\mathrm{N} 2$ are referred to the retinal potentials, the potentials from the retina to the brainstem, and those from the brainstem to the visual cortex, respectively. The flash VEP is an available technique to evaluate the function of visual pathway in dogs.

ACKNOWLEDGEMENT. This work supported in part by 
a Grant-in-Aid for Scientific Research from Ministry of Education, Culture, Sports, Science and Technology of Japan (Nos. 14360189 and 16380211).

\section{REFERENCES}

1. Bichsel, P., Oliver, J. E. Jr., Coulter, D. B. and Brown, J. 1988. Recording of visual-evoked potentials in dogs with scalp electrodes. J. Vet. Int. Med. 2: 145-149.

2. Howard, D. R. and Breazile, J. E. 1973. Optic fiber projections to dorsal lateral geniculate nucleus in the dog. Am. J. Vet. Res. 34: 419-424.

3. Hughes, J. R., Kuruvilla, A. and Fino, J. J. 1992. Topographic analysis of visual evoked potentials from flash and pattern reversal stimuli: evidence for "travelling waves". Brain Topogr. 4: 215-228.

4 Jasper, H. H. 1958. The ten twenty electrode system of the international federation. Electroencephalogr. Clin. Neurophysiol. 10: 371-375.

5. Malnati, G. A., Marshall, A. E. and Coulter, D. B. 1981. Electroretinographic components of the canine visual evoked response. Am. J. Vet. Res. 42: 159-163.

6. Sato, S., Sugimoto, S. and Chiba, S. 1982. A procedure for recording electroretinogram and visual evoked potential in conscious dogs. J. Pharmacol. Methods 8: 173-181.

7. Seri, S., Cerquiglini, A., Cusmai, R. and Curatolo, P. 1991.
Tuberous sclerosis: relationships between topographic mapping of EEG, VEPs and MRI findings. Neurophysiol. Clin. 21: $161-172$.

8. Shimoyama, I., Uemura, K., Morita, Y., Miyanaga, F., Kuroda, R. and Nakamura, T. 1996. Visual evoked potentials to a faint light: signal propagation analyzed with peak latency and topographic mapping. Brain Topogr. 8: 245-247.

9. Sims, M. H., Laratta, L. J., Bubb, W. J. and Morgan, R. V. 1989. Waveform analysis and reproducibility of visual-evoked potentials in dogs. Am. J. Vet. Res. 50: 1823-1828.

10. Strain, G. M., Jackson, R. M. and Tedford, B. L. 1990. Visual evoked potentials in the clinically normal dog. J. Vet. Int. Med. 4: $222-225$.

11. Strain, G. M., Jackson R. M. and Tedford, B. L. 1991. Postnatal development of the visual-evoked potential in dogs. Am. J. Vet. Res. 52: 231-235.

12. Tsutsui, J. and Kawashima, S. 1985. Dynamic topography of the human short latency visual evoked potentials. Neuro-ophthalmology 5: 155-160.

13. Tsutsui, J. and Kawashima, S. 1986. Studies on short latency visual evoked potentials in cases with optic pathway lesions. Neuro-ophthalmology 6: 247-255.

14. Visser, S.L., Stam, F.C., van Tilburg, W., Op den Velde, W., Blom, J.L. and de Rijke, W. 1976. Visual evoked response in senile and presenile dementia. Electroencephalogr. Clin. Neurophysiol. 40: 385-392. 